\title{
Role of Diffusion Weighted MRI in Evaluation of Urinary Bladder Cancer in Iraqi Patient in Correlation with Histopathological Grade.
}

\author{
Dr. Khaleel Ibraheem Mohson, MBChB, CABMS(Rad) ${ }^{1}$, Dr. Mohammed \\ Bassil Ismail, $\mathrm{MBChB}^{2}$, CABMS(uro), Dr. Sura Tami Abduljabbar , $\mathrm{MBChB}^{3}$, \\ ${ }^{I}$ Iraqi national cancer research center / Baghdad University \\ ${ }^{2}$ Urology department, surgical subspeciality hospital, Baghdad medical city complex \\ ${ }^{3}$ Histopathology department, surgical subspeciality hospital, Baghdad medical city complex
}

\begin{abstract}
Background: Bladder cancer is a common urologic cancer that has the highest recurrence rate of any malignancy. MRI (magnetic resonance imaging) is a vital complementary diagnostic device in the diagnosis, staging, and follow up of bladder cancer.

Objectives: The main purpose of this study was to explore the diagnostic effectiveness of pelvic MRI including $A D C$ (apparent diffusion coefficient) and DWI (diffusion weighted imaging) in the assessment of bladder cancer grade and thus helping in patients' management.

Methods: A cross sectional study comprised forty preoperative patients complaining of hematuria referred to the urology consulting clinic in martyr Ghazi Al-Hariri hospital in Baghdad during the period from January 2015 to September 2016. Their age ranged from 43 to 74 years. Their MRI findings were reviewed and correlated with the corresponding histopathology results.

Results:By reviewing the MRI finding in forty patients, we see that $60 \%$ show low grade tumor, while $40 \%$ had high grade tumor this is classified according to their ADC map which revealed that all patients with high grade tumor had their tumor is above $9 \times 10^{-3} \mathrm{~mm}^{2} /$ second while those with low grade tumor had value greater than above value, wall invasion on T2WI ( T2 weighted imaging) is seen in 33\% of low grade tumor and $81 \%$ of high grade tumor, in contrary to DWI that identify wall invasion in $21 \%$ and $43 \%$ of the tumors respectively.

Conclusion: $A D C$ map and DWI using b-value of 0 and 1000 respectively are of greater value in localization, evaluation and prediction of histological grade of bladder cancer.
\end{abstract}

Keywords: diffusion weighted MRI, apparent diffusion Coefficient, urinary bladder cancer, histopathological grade.

\section{Introduction}

Urothelial carcinomas are mainly located in the bladder and to lesser extend in upper urinary tract including renal collecting system and ureters, Bladder cancer account for 90-95\% of urothelial carcinoma.[1,2]Bladder cancer is a frequent malignancy of the lower urinary tract, showing an incidence of 10.1 per 100,000 for men and 2.5 per 100,000 for women [3].Transurethral resection of the bladder tumor (TURBT) is the standard treatment for non-muscle-invasive cancer, by which the urologist should provide the pathologist complete sampling of the tumor, the underlying bladder wall, and the edges of the resection [4]. The pathologist should report specifically the histology type, the grade, the depth of lamina propria invasion, and the presence of muscle in the specimen [5].

Bladder cancer also may show divergent histopathology, of which $90 \%$ of cases in the United States and western Europe presenting is urothelial carcinoma [either as pure transitional cell carcinoma (TCC) or as TCC with focal squamous or glandular differentiation] and $3 \%$ to $6 \%$ as pure squamous cell carcinoma (SCC)[6]. In recent clinical practice, contrast-enhanced computed tomography and MRI have become the most widely-used imaging modalities for radiological evaluation of the upper urinary tract and bladder. [7]MRI is superior to computed tomography in loco regionalstaging because of its superior soft tissue delineation. [8, 9]DW-MRI is a type of functional imaging, which is increasingly being applied in the detection ,characterization and staging of bladder cancer, also provides helpful information for the diagnosis of urothelial carcinoma in a non-invasive manner.[10,11]Growing evidence has emerged showing that DW-MRI can serve as an imaging biomarker for characterizing the pathophysiology of cancer.[8]in DWI malignant lesions characteristically show high signal intensity because of the dense cellularity, tissue disorganization and decreased extracellular space that are typical characteristics of cancerous tissue, all of which restrict water diffusion.[11,12]On DW-MRI with high $b$-values of $800-1000 \mathrm{~s} / \mathrm{mm}^{2}$, urothelial carcinomas in the upper urinary tract and bladder generally show a homogenous, hyperintense signal reflecting homogenous histological 
characteristics, whereas the signals from the surrounding tissues, including the urine, renal parenchyma and surrounding tissues, are well-restrained.[13]

\section{Patients and methods}

This is a cross sectional study that comprised forty preoperative patients complaining of hematuria referred to the urology consulting clinic in martyr Ghazi Al-Hariri hospital in the medical city complex in Baghdad during the period from January 2015 to September 2016, their age ranged from 43 to 74 years. All patients were subjected to pelvic MRI performed by Philips 1.5 tesla machine using T1, T2, fat suppression, ADC map and DW ( $b$-value1000) sequences, in axial, coronal and sagittal planes ,except DWI was done in axial plane. Patients were examined in supine position with full bladder state before and after intravenous gadolinium injection. The presence or absence of mass lesion were assessed: including their position and wall invasion, then ADC map was calculated for each patient, ADC map or histogram is calculated by drawing a $20 \mathrm{~mm}^{2}$ area in the center of tumor using $b$-value 0 and histogram is displayed showing $\mathrm{x}$ and y axes, the mean ADC value is shown in form of $\left(10^{-3} \mathrm{~mm}^{2} / \mathrm{second}\right)$.

All patient underwent transurethral resection of bladder tumors; whether under general or spinal anesthesia. In the operation room, the patient is placed in dorsal lithotomy position, then a thorough endoscopic evaluation of the urethra, prostate (in male), and bladder is done. The bladder is filled with $1.5 \%$ glycine irrigant; once the tumor is identified, using resectoscope, deep resection (near perforation) using a cutting current on the loop resection, then sufficient tissue is sent for histopathology.

The MRI findings were correlated with the corresponding histopathological results.

Exclusion criteria:

1. Patient with extravesicle primary pelvis cancer

2. Patients previously diagnosed to have bladder cancer and referred for follow up.

\section{Results}

Forty patients presenting with hematuria were included in this study. Their age were ranged between 43 and 74 years (mean 58 years).

Figure 1 and 2

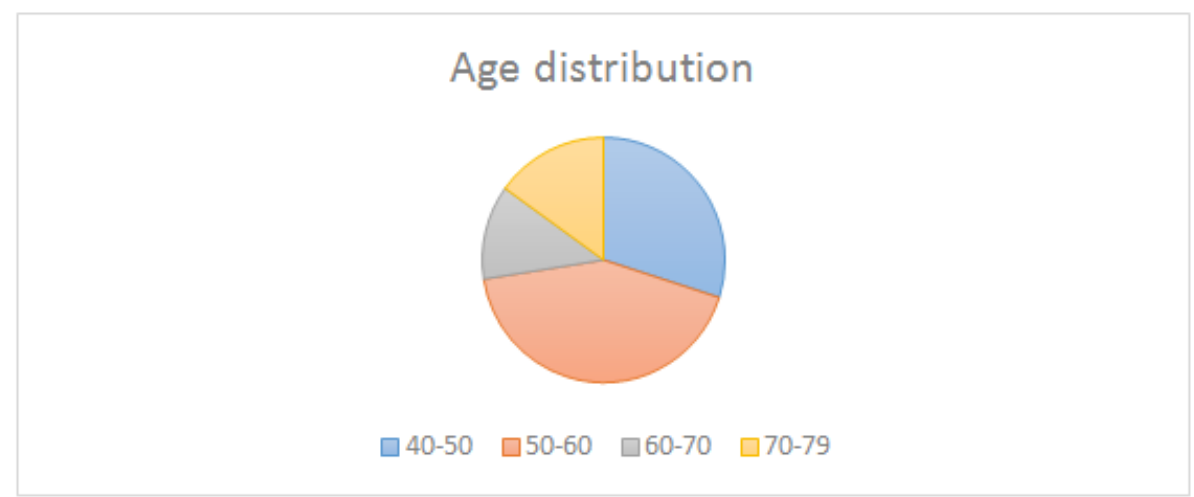

Figure 1 age distribution: most patients are in $6^{\text {th }}$ decade

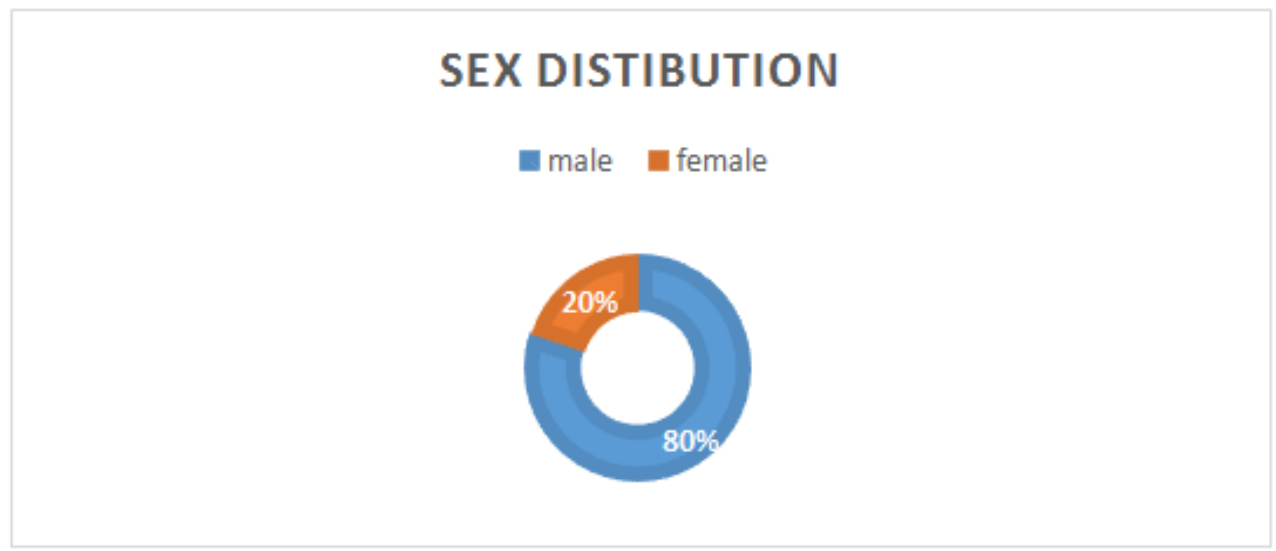

Figure 2: sex distribution: 
Low ADC value (below $9 \times 10^{-3} \mathrm{~mm}^{2} / \mathrm{second}$ ) is registered in high grade tumor and this will represent $40 \%$ of preoperative patients, while low grade tumor value $\left(10 \times 10^{-3} \mathrm{~mm}^{2} / \mathrm{second}\right)$ is seen in 16 preoperative patients ,DWI using $b$-value 1000 shows restricted diffusion -high Signal- in both in comparison to urine. Correlation between ADC map and histopathological grade are displayed in table 1

Table 1: ADC map results in correlation with tumor grade

\begin{tabular}{|l|l|l|}
\hline ADC map & Low grade & High grade \\
\hline Low ADC value & $24 / 40(60 \%)$ & 0 \\
\hline High ADC value & 0 & $16 / 40(40 \%)$ \\
\hline
\end{tabular}

Now regarding wall invasion detected during the study by comparing DWI to T2WI: As the DWI is a functional study depending on water particles movement, for this it has lower detection capability than structural T2WI for maroscopical muscle invasion and perivesical extension, by that only $21 \%$ of low grade tumor and $43 \%$ of high grade lesion shows wall invasion by DWI while the reality is $33 \%$ in low grade tumor and $81 \%$ of high grade tumor had muscle invasion by analyzing T2WI and when comparing that with histopathological stage.

Table 2: Macroscopical muscular wall invasion DWI Versus T2WI in comparison with histopathology

\begin{tabular}{|l|l|l|}
\hline Wall invasion & Low grade tumor & High grade tumor \\
\hline T2W & $8 / 24(33 \%)$ & $13 / 16(81 \%)$ \\
\hline DWI & $5 / 24(21 \%)$ & $9 / 16(56 \%)$ \\
\hline
\end{tabular}

\section{Discussion}

Bladder carcinoma is a heterogeneous neoplasm, presenting as either superficial disease $(80 \%)$ or muscle-invasive disease (20\%). Only $10 \%$ to $30 \%$ of superficial tumors (pTa and pT1) will progress to invasive disease, whereas muscle-invasive tumors (pT2-pT4) have a much less favorable prognosis.Sothe more advanced the diagnostic modality is the more helpful it will to determine whether patients have bladder cancer that can be treated locally or requires more aggressive, surgical treatment [14-18].

The advances in MRI examination and the introduction of DWI is considered a great improvement for disease diagnosis and follow up ,DWI is one of the technique and as it's a functional study depends on movement of water particles, A lesion where water molecules can diffuse freely shows low-signal intensity on DW-MRI and reverse is true ,thus DW-MRI has been applied in the early detection of microstructural and functional changes.[19]

In our study the bladder tumor in general had restriction in their water movement, so reveals high signal on DWI ,for that DWI is best for tiny tumor localization, while the ADC map used to characterize its value using the histogram and thus can determine its grade by correlation the value with histopathological results, tumor with low ADC value is considered high grade while those with high value are considered low and this is in agree with study that was done by Takeuchi et al. reported that the ADC values of grade 3 bladder cancers were significantly lower than those of grade 1 or 2 bladder cancers. [20] Regarding the macroscopical wall invasion, still T2WI better than DWI in assessment of focal wall and extravesicle extension as the T2WI is a structural image where muscular wall is hypointense and tumor is hyperintense so wall invasion is clarify ,while DWI is functional imaging and can to lesser degree assess wall invasion by depending the difference in restriction between the restricted tumor and non-restricted normal wall. From the above results we can use DWI alone with no need of intravenous gadolinium contrast media especially in tired elderly with impaired renal indices. MRI is often described as a "safe" modality due to the fact that, unlike x-ray based modalities, ionizing radiation is not encountered. However, there are hazards intrinsic to the MR environment that must be acknowledged and excluded. Most reported cases of MR related injuries and the few fatalities that have occurred have apparently been the result of failure to follow safety guidelines or have resulted from the use of inappropriate or outdated information related to the safety aspects of biomedical implants and devices.[21]

\section{Conclusion}

MRI is an efficient, safe tool in diagnosing, grading and staging of urothelial cancer, specifically when superadded by using DWI and when accompanied by thorough and competent pathological results. It is thus recommended as an initial investigation in any patient presenting with bladder mass.

\section{References}

[1]. 1.Babjuk M, Oosterlinck W, Sylvester R et al. EAU guidelines on non-muscle-invasive urothelial carcinoma of the bladder, the 2011 update. Eur. Urol. 2011; 59: 997-1008.

[2]. Roupret M, Zigeuner R, Palou J et al. European guidelines for the diagnosis and management of upper urinary tract urothelial cell carcinomas: 2011 update. Eur. Urol. 2011; 59: 584-94. 
[3]. Ploeg M, Aben KK, Kiemeney LA. The present and future burdenof urinary bladder cancer in the world.World J Urol. 2009; 27:289-93.

[4]. Babjuk M, Burger M, Zigeuner R, et al. EAU guidelines on nonmuscle-invasive urothelial carcinoma of the bladder: update 2013.Eur Urol. 2013; 64:639-53.

[5]. Ramírez-Backhaus M, Domínguez-Escrig J, Collado A, et al.Restaging Transurethral Resection of Bladder Tumor for HighriskStage Ta and T1 Bladder Cancer. CurrUrol Rep. 2012; 13:109-14.

[6]. Johnson DE,SchoenwaldMB, Ayala AG, et al. Squamouscell carcinoma of the bladder. J Urol 1976;115:542^4.

[7]. Green DA, Durand M, Gumpeni N et al. Role of magnetic resonance imaging in bladder cancer: current status and emerging techniques. BJU Int. 2012;110: 1463-70.

[8]. 8.Kamat AM, Hegarty PK, Gee JR et al. ICUD-EAU International Consultation on Bladder Cancer 2012: screening, diagnosis, and molecular markers. Eur.Urol. 2013; 63: 4-15.

[9]. Raza SA, Jhaveri KS. MR imaging of urinary bladder carcinoma and beyond. Radiol. Clin. North Am. 2012; 50: 1085-110.

[10]. Giannarini G, Petralia G, Thoeny HC. Potential and limitations of diffusion-weighted magnetic resonance imaging in kidney, prostate, and bladder cancer including pelvic lymph node staging: a critical analysis of the literature. Eur. Urol. 2012; 61: 326-40.

[11]. 11.Koh DM, Collins DJ. Diffusion-weighted MRI in the body: applications and challenges in oncology. AJR Am. J. Roentgenol. 2007; 188: 1622-35.

[12]. Padhani AR, Liu G, Koh DM et al. Diffusion-weighted magnetic resonance imaging as a cancer biomarker: consensus and recommendations. Neoplasia 2009; 11: 102-25.

[13]. Takeuchi M, Sasaki S, Naiki T et al. MR imaging of urinary bladder cancer for T-staging: a review and a pictorial essay of diffusion-weighted imaging.J. Magn. Reson. Imaging 2013; 38: 1299-309.

[14]. Knowles MA. The genetics of transitional cell carcinoma:progress and potential clinical application.BJUInt1999;84:412-27.

[15]. SpruckCHIII,Ohneseit PF, Gonzalez-ZuluetaM, et al.Two molecular pathways to transitional cell carcinomaof the bladder. Cancer Res1994;54:784- 8.

[16]. Simoneau AR, Jones PA. Bladder cancer: themolecularprogression to invasive disease. WorldJUrol1994;12:89 - 95.

[17]. Dalbagni G, Presti J, Reuter V, FairWR, Cordon-Cardo C. Genetic alterations in bladder cancer. Lancet1993;342:469-71.

[18]. Cordon-Cardo C, Sheinfeld J, Dalbagni G. Geneticstudies and molecular markers of bladder cancer.Semin SurgOncol1997;13:31927.

[19]. Le Bihan D, Breton E, Lallemand D, Grenier P, Cabanis E, Laval-Jeantet M. MR imaging of intravoxel incoherent motions: application to diffusion and perfusion in neurologic disorders. Radiology 1986; 161: 401-7.

[20]. 20.Takeuchi M, Sasaki S, Ito M et al. Urinary bladder cancer: diffusion-weighted MR imaging - accuracy for diagnosing T stage and estimating histologic grade. Radiology 2009; 251: 112-21.

[21]. 21.Shellock FG. Reference manual for magnetic resonance safety,implants, and devices, 2006 ed. Los Angeles: Biomedical ResearchPublishing, 2006. 